\title{
Descrição anatômica dos ramos colaterais do arco aórtico do tamanduá-mirim (Tamandua tetradactyla)
}

\author{
Valdelice de Lourdes Corrêa Pinheiro ${ }^{1}$ \\ Ana Rita de Lima ${ }^{1}$ \\ Luiza Corrêa Pereira ${ }^{2}$ \\ Bruno Duarte Gomes ${ }^{3}$ \\ Érika Branco ${ }^{1 *}$ \\ ${ }^{1}$ Universidade Federal Rural da Amazônia \\ Avenida Presidente Tancredo Neves, 2501, CEP 66077-530, Belém - PA, Brasil \\ ${ }^{2}$ Área de Mina Bauxita, Vale Paragominas - PA, Brasil \\ ${ }^{3}$ Instituto de Ciências Biológicas, Universidade Federal do Pará, Belém - PA, Brasil \\ * Autor para correspondência \\ ebranco.ufra@gmail.com
}

\section{Resumo}

Com o objetivo de ampliar os estudos e conhecimentos anatômicos, bem como impulsionar novas pesquisas com animais selvagens, estudaram-se três exemplares de tamanduá-mirim (Tamandua tetradactyla), atropelados, sendo, dois machos e uma fêmea, provenientes de Paragominas-PA, sob autorizações SEMA-PA n. 455/2009 e 522/2009, doados à Universidade Federal Rural da Amazônia (UFRA) após óbito por causas naturais. O sistema arterial foi preenchido com látex corado em vermelho e os animais foram fixados e dissecados para observação da disposição dos ramos colaterais do arco aórtico. Inicialmente, destacamos o tronco braquiocefálico foi identificado, de onde tiveram origem a artéria subclávia direita e o tronco bicarotídeo, o qual se bifurcou em artéria carótida comum direita e esquerda. O segundo ramo originado no arco aórtico foi a artéria subclávia esquerda, que se ramificou em artéria vertebral, artéria torácica interna, tronco costocervical e artéria cervical superficial. Esses mesmos ramos também estiveram presentes na artéria subclávia direita, a qual, em nível axilar, passou a ser chamada de artéria axilar. De modo geral, observou-se que os três tamanduás-mirins estudados apresentaram padrão vascular distinto de outros mamíferos já estudados, incluindo espécies que compartilharam a superordem dos Xenartras, sugerindo que nessa espécie possa haver um padrão particular de distribuição dos principais ramos oriundos do arco aórtico.

Palavras-chave: Arco aórtico; Ramos colaterais; Tamanduá-mirim

\section{Abstract}

Anatomic description of the collateral branches from the aortic arch of lesser anteater (Tamandua tetradactyla). Aiming to enrich the anatomical study and knowledge and foster new researches with wild animals, three runned over lesser anteaters (Tamandua tetradactyla) were studied, being two males and one female, from Paragominas, Para, Brazil, under the licenses SEMA-PA 455/2009 and 522/2009, which were 
donated to Universidade Federal Rural da Amazônia (UFRA) after death due to natural causes. The arterial system was filled with red latex and the animals were fixed and dissected for observation of the arrangement of the collateral branches of the aortic arch. First, the brachiocephalic trunk was identified, from which originated the right subclavian artery and the bicarotid trunk, which bifurcated into right and left common carotid artery. The second branch originated in the aortic arch was the left subclavian artery, which branched into the vertebral artery, internal thoracic artery, costocervical trunk, and superficial cervical artery. These same branches were also present in the right subclavian artery, which, in the axillary level, was named the axillary artery. In general, it was observed that the three lesser anteaters studied presented a different vascular pattern from others mammals already studied, including species from the Xenarthra superorder, suggesting that in this species there might exist a particular pattern of distribution of the main branches from the aortic arch.

Key words: Aortic arch; Collateral branches; Lesser anteater

\section{Introdução}

O tamanduá-mirim (Tamandua tetradactila) é um mamífero euteriano, pertencente à ordem Edentata, Superordem Xenarthra, assim como o tatu e a preguiça sendo normalmente encontrado da Venezuela ao Sul do Brasil. Possui cabeça, membros e porção cranial do dorso com pelagem de coloração amarelada, sendo o restante do corpo com pelagem negra, formando uma espécie de colete. A cauda longa é preênsil e os membros torácicos possuem quatro grandes garras. Com hábito predominantemente noturno, os tamanduás-mirins são animais solitários, silenciosos e de índole pacífica. Em vida livre, são vistos ou sempre sozinhos, ou juntos em época de acasalamento ou com sua cria, porém, em cativeiro, aceitam viver conjuntamente. A base alimentar desses animais é composta basicamente por insetos, principalmente formigas e cupins (CUBAS et al., 2007).

No que tange a aorta, objeto alvo deste estudo, esta tem origem no ventrículo esquerdo sendo o principal tronco sistêmico. Dirige-se para a direita e para cima dentro do mediastino médio, constituindo a aorta ascendente. Curva-se para a esquerda e para trás, adentrando no mediastino superior e formando o arco aórtico (GUSMÃO, 2003).

Nos mamíferos domésticos, a aorta ascendente alcança em seu curso, a coluna vertebral ao nível da $6^{\mathrm{a}}$ vértebra torácica, um pouco à esquerda do plano mediano, no mediastino médio, prosseguindo como aorta descendente em direção caudal. Na cavidade torácica, torna-se aorta torácica, e na cavidade abdominal, é denominada aorta abdominal. Através de uma abertura no diafragma, o hiato aórtico, passa para a cavidade abdominal. Sua divisão final ocorre ao nível da última vértebra lombar (GETTY, 1981).

De forma geral, o arco aórtico origina um único grande tronco braquiocefálico, cujas ramificações distribuem sangue para a metade cranial do animal. O padrão exato das principais ramificações arteriais depende das espécies, porém, via de regra, elenca-se as artérias subclávias, troncos costocerviais e artérias carótidas comuns, as quais se originam do tronco bicarotídeo (FRANDSON et al., 2005).

$\mathrm{O}$ tamanduá-mirim pertence a um dos grupos de animais selvagens menos estudados do ponto de vista morfológico. Desta forma, o presente estudo tem como objetivo descrever as disposições dos ramos colaterais do arco aórtico buscando dar suporte ao estudo da anatomia comparada.

\section{Material e Métodos}

Foram estudados quatro animais jovens (média Crown-rump length (CRL) de 41,5cm), sendo dois machos e duas fêmeas, provenientes de Paragominas PA, Brasil/Empresa Terra LTDA, sob autorização SEMA - PA no 455/2009 e 522/2009, doados ao Instituto de Saúde e Produção Animal da Universidade Federal Rural da Amazônia - UFRA após óbito por atropelamento. O sistema vascular arterial foi preenchido com látex corado em vermelho adicionado de contraste radiográfico baritado na proporçao de 1:1, a partir da canulação da aorta torácica. Os animais foram fixados por aplicações intramusculares, subcutâneas e intracavitárias de 
solução aquosa de formaldeído $10 \%$. Os animais foram radiografados e dissecados para identificação dos ramos colaterais do arco aórtico.

Toda nomenclatura adotada foi baseada na Nomina Anatomica Veterinaria (INTERNATIONAL COMMITTEE ON VETERINARY GROSS ANATOMICAL NOMENCLATURE, 2005).

\section{Resultados}

Imediatamente após a origem do arco aórtico no tamanduá-mirim, observou-se o tronco braquiocefálico, constituindo o primeiro ramo colateral, e deste originouse a artéria subclávia direita e o tronco bicarotídeo, de onde surgiram as artérias carótidas comum direita e esquerda.

A artéria subclávia direita ramificou-se em: artéria vertebral, artéria torácica interna, tronco costocervical e artéria cervical superficial. Por sua vez, a artéria subclávia esquerda derivou diretamente do arco aórtico, constituindo o segundo ramo colateral deste, imediatamente após o tronco bicarotídeo. Esta artéria projetou-se levemente à esquerda até ultrapassar cranialmente a primeira costela. Apesar da origem diferente, a artéria subclávia esquerda apresentou os mesmos ramos já descritos para a artéria subclávia direita, ou seja, artéria torácica interna, tronco costocervical, artéria vertebral, e artéria cervical superficial. Após a emissão desses ramos, ambas as artérias subclávias, alcançaram o espaço axilar, possuindo um calibre maior em relação a seus ramos, recebendo o nome de artéria axilar direita e esquerda (Figura $1 \mathrm{~A}-\mathrm{C}$ ).

A artéria torácica interna do tamanduá-mirim foi o primeiro ramo a surgir das artérias subclávias, seguido do tronco costocervical, artéria vertebral e artéria cervical superficial.

Nesta espécie, as artérias carótidas comum direita e esquerda se ramificaram para vascularizar o músculo esternoióideo, que teve origem no osso hióide e inserção na cartilagem xifóide do esterno, acompanhando ventralmente toda a região cervical e torácica. Essas ramificações se inseriram no interior da faixa desse músculo, percorrendo-o internamente (Figura 1B).

\section{Discussão}

Devido à escassez de literatura abordando os aspectos morfológicos de xenartras em geral, pouco se sabe sobre a anatomia destes animais, porém, a literatura conta com o relato da descrição do arco aórtico em tatus galinha (DOMENICONI et al., 2004), descrevendo a formação de dois troncos braquiocefálicos, sendo eles direito e esquerdo emergindo do arco aórtico. Desses troncos, nessa espécie, são originadas as artérias carótidas comuns e as artérias subclávias, direita e esquerda, respectivamente. Porém, em tamanduá-mirim, que também é um xenartra, encontramos apenas uma artéria braquiocefálica, a qual emitiu os mesmos ramos citados pelos autores supramencionados. Tal achado vai de encontro com o que foi descrito por Goshal (1981) em relação aos cães. No entanto, Culau et al. (2004) observaram serem possíveis variações dessa conformação, não só em cães, no qual o arco aórtico emitiu o tronco bicarotídeo e as artérias subclávias, direita e esquerda, não apresentando artéria braquiocefálica, diferentemente do que foi relatado por Cortellini et al. (2002) em búfalos, que não só não apresentaram o tronco bicarotídeo como também em alguns animais estudados o tronco braquiocefálico originou a artéria subclávia esquerda em comum ao tronco costocervical esquerdo.

Mantendo essa linha investigativa quanto a grande variação que ocorre no arco aórtico, Culau et al. (2007), em capivaras, relatam que em 100\% dos casos foi observado um único ramo colateral do arco aórtico, sendo este o tronco braquiocefálico, o qual apresentou variações revelando que em $57,1 \%$ dos animais estudados a artéria subclávia esquerda, carótida comum esquerda e tronco bicarotídeo, originaram-se do tronco braquiocefálico. As artérias carótida comum direita e subclávia direita foram lançadas pelo tronco bicarotídeo. No entanto, em $28,6 \%$ das capivaras, o tronco braquiocefálico originou primeiramente a artéria subclávia esquerda, que então se trifurcou em artéria subclávia direita, artéria carótida comum direita e artéria carótida comum esquerda. E por último, em 14,3\% dos casos, originaram-se do tronco braquiocefálico primeiramente a artéria subclávia esquerda, logo após a artéria subclávia direita e em seguida, um tronco bicarotídeo, que emitiu as artérias carótidas comuns 
FIGURA 1: (A) Ramos colaterais do arco aortico do Tamandua tetradactyla. 1 - arco aórtico, 2 - tronco braquiocefálico, 3 - artéria carótida comum direita e esquerda, 4 - artéria subclávia direita e esquerda, 5 - artéria torácica interna direita e esquerda, 6 - artéria cervical superficial direita e esquerda, 7 - artéria vertebral direita e esquerda, 8 - tronco costocervical direito e esquerdo; 9 - artéria axilar direita e esquerda; (B) 10 - arco aórtico, 11-tronco bicarotídeo, 12 - artéria carótida comum esquerda,13 -artéria carótida comum direita, 14 - artéria subclávia esquerda, 15 - artéria subclávia direita, 16 - artéria mamária interna esquerda. Notar ramo da artéria carótida comum esquerda irrigando o músculo esternoiódeo (seta), (C) Imagem radiográfica do Tamandua tetradactyla. Notar os ramos colaterais do arco aórtico. 17 - arco aórtico, 18 - tronco bicarotídeo, 19 - artéria carótida comum direita e esquerda, 20 - artéria subclávia direita e esquerda.
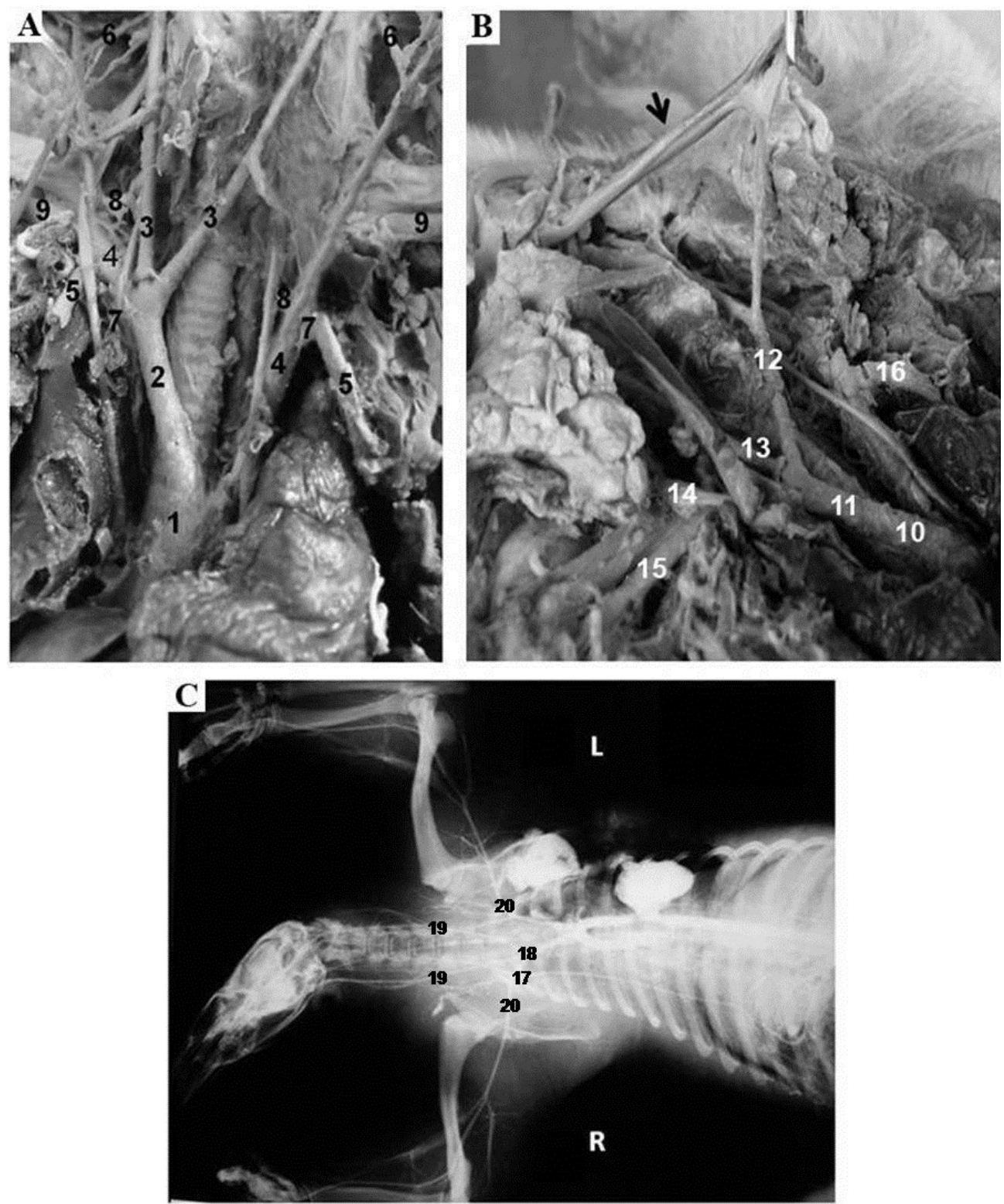
direita e esquerda. No T. tetradactyla não foi observada essa variação de disposição dos ramos do arco aórtico, de forma que se destacaram apenas dois ramos, sendo eles o tronco braquiocefálico e artéria subclávia esquerda.

No que tange as artérias subclávias, Domeniconi et al. (2004) relataram que em tatus galinha estes vasos originaram apenas as artérias omocervicais e as axilares, diferente do que encontramos nos tamanduás-mirins estudados, no qual notamos a presença das artérias torácica interna, tronco costocervical, artéria vertebral e artéria cervical superficial, corroborando com os achados de Santos et al. (2004), em mão-pelada.

De forma mais complexa, Araújo et al. (2004) descreveram que em chinchilas a artéria subclávia direita originou ramos colaterais sequenciais como artérias vertebral, escapular dorsal, tronco comum torácica interna-vertebral torácica e tronco comum cervical superficialprofunda, enquanto, à esquerda, originou, o tronco comum torácica interna-vertebral torácica.

Diante do exposto, conclui-se que os três tamanduásmirins estudados apresentaram padrão vascular distinto de outros mamíferos já estudados, incluindo espécies que compartilharam a Superordem dos xenartras, sugerindo que nesta espécie possa haver um padrão particular de distribuição dos principais ramos oriundos do arco aórtico.

\section{Referências}

ARAÚJO, A. C. P.; OLIVEIRA, J. C. D.; CAMPOS, R. Ramos colaterais do arco aórtico e as suas principais ramificações em chinchilas (Chinchilla lanigera). Revista Portuguesa de Ciências Veterinárias, Lisboa, v. 99, p. 53-58, 2004.
CORTELLINI, L. M. F.; MACHADO, M. R. F.; OLIVEIRA, F. C.; MIGLINO, M. A.; ARTONI, S. M. B. Ramos do arco aórtico de bubalinos. Ciência Rural, Santa Maria, v. 30, p. 445-448, 2000.

CUBAS, Z. S.; SILVA, J. C. R.; CATÃO-DIAS, J. L. Tratado de animais selvagens - Medicina Veterinária. 1 ed. São Paulo: Roca, 2007. $1354 \mathrm{p}$.

CULAU, P. O. V.; OLIVEIRA, J. C. D.; RECKZIEGEL, S. H.; LINDEMANN, T. Origem ectópica da artéria subclávia direita e do tronco bicarotídeo no cão. Ciência Rural, Santa Maria, v. 34, p. 1615-1618, 2004.

CULAU, P. O. V.; RECKZIEGEL, S. H.; LINDEMANN, T.; ARAÚJO, A. C. P.; BALZARETTI, F. Colaterais do arco aórtico da capivara (Hidrochoerus hidrochaeris). Acta Scientiae Veterinariae, Porto Alegre, v. 35, p. 89-92, 2007.

DOMENICONI, R. F.; ABREU, M. A. F.; BENETTI, E. J.; VILAÇA, J. S. The contribution of the aortic branches in the vascularization of cervical regions, during the development of the nine banded armadillo (Dasypus Novemcinctus, L.1758). International Journal of Morphology, Temuco, v. 22, p. 113-118, 2004.

FRANDSON, R. D.; WILKE, W. L.; FAILS, A. D. Anatomia e fisiologia dos animais de fazenda. Rio de Janeiro: Guanabara Koogan, 2005. 454 p.

GETTY, R. Anatomia dos animais domésticos. 5 ed. Rio de Janeiro: Guanabara Koogan, 1986. 2000 p.

GHOSHAL, N. 1986. Coração e artérias. In: GETTY, R. (Ed.). Anatomia dos animais domésticos. 5. ed. Rio de Janeiro: Guanabara Koogan, 1986. p. 528-529.

GUSMÃO, L. Anatomia arterial venosa e aplicada. In: PITTA, G. B. B.; CASTRO, A. A.; BURIHAN, E. (eds). Angiologia e cirurgia vascular: guia ilustrado. Maceió: UNCISAL/ECMAL \& LAVA, 2003. p. 1-9.

INTERNATIONAL COMMITTEE ON VETERINARY GROSS ANATOMICAL NOMENCLATURE. Nomina anatômica veterinária. 5 ed. Knoxville: World Association on Veterinary Anatomist, 2005. $190 \mathrm{p}$

SANTOS, A. L. Q.; MORAES, F. M.; MALTA, T. S.; CARVALHO, F. S. M.; ALVES JÚNIOR, J. R. F. Topografia dos colaterais calibrosos do arco aórtico de um mão-pelada (Proncyon cancrivorus - Gray, 1865), (Carnivora-Proncyoniadae). Archives of Veterinary Science, Curitiba, v. 9, p. 67-72, 2004. 